\title{
INVERSE PROBLEMS FOR A DEGENERATE MIXED PARABOLIC-HYPERBOLIC EQUATION ON FINDING TIME-DEPENDING FACTORS IN RIGHT HAND SIDES
}

\author{
S.N. SIDOROV
}

\begin{abstract}
We consider direct and inverse problems on determining time-dependent factors in the right hand sides for a mixed parabolic-hyperbolic equation with a degenerate hyperbolic part in a rectangular area. As a preliminary, we study a direct initial boundary problem for this equation. By the method of spectral analysis we establish the uniqueness criterion for the solution and the solution is constructed as a sum over the system of the eigenfunctions of the corresponding one-dimensional Sturm-Liouville spectral problem. In justifying the convergence of the series, the problem of small denominators arises. Because of this, we prove the estimates for the distance from the zero to the small denominators with a corresponding asymptotics. These estimates allow us to justify the convergence of the constructed series in the class of regular solutions of this equation. On the base of the solution to the direct problem, we formulate and study three inverse problems on finding time-dependent factors in the right hand side only by the parabolic or hyperbolic part of the equation, and also as the factors in the both sides of the equation are unknown. Using the formula of solution to the direct initial boundary problem, the solution of inverse problems is equivalently reduced to the solvability of loaded integral equations. By means of the theory of integral equations, the corresponding theorems of uniqueness and the existence of solutions of the stated inverse problems are proved. At that, the solutions of inverse problems are constructed explicitly, as sums of orthogonal series.
\end{abstract}

Keywords: equation of mixed parabolic-hyperbolic type, initial boundary value problem, inverse problems, uniqueness, existence, series, small denominators, integral equations.

Mathematics Subjects Classifications: 35M10 + 35R30

\section{Formulation of Problem}

We consider a mixed type equation

$$
L u=F(x, t)
$$

in a rectangular domain

$$
D=\{(x, t) \mid 0<x<l,-\alpha<t<\beta\},
$$

where $m>0, l>0, \alpha>0, \beta>0$ are given real numbers and $b$ is a prescribed real number, and

$$
L u=\left\{\begin{array}{l}
u_{t}-u_{x x}+b u, \\
(-t)^{m} u_{x x}-u_{t t}-b(-t)^{m} u,
\end{array} \quad F(x, t)= \begin{cases}f_{1}(x) g_{1}(t), & t>0 \\
f_{2}(x) g_{2}(t), & t<0\end{cases}\right.
$$

We pose the following problems.

S.N. Sidorov, InvERSE PROBlems FOR A DEGENERATE MIXED PARABOLIC-HYPERboliC EQUATION ON FINDING TIME-DEPENDING FACTORS IN RIGHT HAND SIDES.

(C)Sidorov S.N. 2019.

The reported study was funded by RFBR according to the research project no. 17-41-020516).

Submitted April 7, 2018. 
Problem 1. Find a function $u(x, t)$ satisfying the conditions

$$
\begin{aligned}
& u(x, t) \in C(\bar{D}) \cap C_{t}^{1}(D) \cap C_{x}^{1}(\bar{D}) \cap C_{x}^{2}\left(D_{+}\right) \cap C^{2}\left(D_{-}\right) ; \\
& L u(x, t) \equiv F(x, t), \quad(x, t) \in D_{+} \cup D_{-} ; \\
& u(0, t)=u(l, t)=0, \quad-\alpha \leqslant t \leqslant \beta ; \\
& u(x,-\alpha)=0, \quad 0 \leqslant x \leqslant l,
\end{aligned}
$$

where $F(x, t)$ is a given sufficiently smooth function, $D_{+}=D \cap\{t>0\}, D_{-}=D \cap\{t<0\}$.

Problem 2. Find functions $u(x, t)$ and $g_{1}(t)$ satisfying conditions (1.2)-(1.5) and

$$
\begin{aligned}
& g_{1}(t) \in C[0, \beta] ; \\
& u\left(x_{0}, t\right)=h_{1}(t), \quad 0<x_{0}<l, \quad 0 \leqslant t \leqslant \beta,
\end{aligned}
$$

where $f_{i}(x), i=1,2, g_{2}(t), h_{1}(t)$ are given functions, $x_{0}$ is a given point in the interval $(0, l)$, $D_{+}=D \cap\{t>0\}, D_{-}=D \cap\{t<0\}$.

Problem 3. Find functions $u(x, t)$ and $g_{2}(t)$ satisfying conditions (1.2)-(1.5) and

$$
\begin{aligned}
& g_{2}(t) \in C[-\alpha, 0], \\
& u\left(x_{0}, t\right)=h_{2}(t), \quad 0<x_{0}<l, \quad-\alpha \leqslant t \leqslant 0,
\end{aligned}
$$

where $f_{i}(x), i=1,2, g_{1}(t), h_{2}(t)$ are known functions.

Problem 4. Find functions $u(x, t), g_{1}(t), g_{2}(t)$, satisfying conditions (1.2)-(1.9), where $f_{i}(x), h_{i}(t), i=1,2$, are given functions.

We note that in Problems 2-4 conditions (1.7) and (1.9) are additional for determining the functions $g_{1}(t)$ and $g_{2}(t)$.

One of the first studies of interface problem with a parabolic equation on one part of a domain and a hyperbolic equation on the other part is work by I.M. Gel'fand [1]. He considered an example a gas motion in a channel surrounded by a porous medium and at that, the gas motion in a channel was described by a wave equation, while outside the channel the diffusion equation was posed. Ya.S. Uflyand $[2,3]$ considered a problem on propagation of electric oscillations in compound lines, when the losses on a semi-infinite line were neglected and the rest of the line was treated as a cable with no leaks. He reduced this problem a mixed parabolic-hyperbolic type equation. This problem for a more general equation was considered in monograph by T.D. Dzhuraev [4].

O.A. Ladyzhenskaya and L. Stupjalis $[5,6]$ considered initial boundary value problems in a multi-dimensional space with interface for parabolic-hyperbolic equations arising in studying problem on motion of a conducting liquid in an electromagnetic field.

In works by N.Yu. Kapustin [7], the methods of functional analysis were employed for proving the unique solvability of an analogue of Tricomi problem in the space $L_{2}$ for equation (1.1) as $b=0,0<m \leqslant 1$ and $F(x, t) \equiv 0$ in a mixed domain, whose parabolic part coincided with $D_{+}$, while the hyperbolic part was a characteristic triangle with a base on the degeneration line. In a similar domain, E.I. Moiseev and N.Yu. Kapustin [8] studied problems for parablic-hyperbolic equations and corresponding one-dimensional spectral problems by using the methods of spectral analysis.

Earlier problems 1-4 we first posed and studied in works by K.B. Sabitov [9], [10] for equation (1.1) with $m=0$. Initial boundary value problem (1.2) (1.5) for homogeneous equation (1.1) with $F_{i}(x, t) \equiv 0, i=1,2$, was studied in work [11-14], while the case $F_{i}(x, t) \neq 0$ was considered in work [15]. In works [16-18] there were studied inverse problems on finding the functions $u(x, t)$ and $f_{i}(x)$ as $g_{i}(t) \equiv 1$.

Inverse problem arise in many fields of natural sciences: electrodynamics, acoustics, quantum scattering theory, geophysics (in inverse problems of electrical exploration, seismicity, potential theory), astronomy and other fields. This relates to the fact that the values of the parameters 
can be obtained from the observed data, while the properties of the medium are often unknown in practice.

Various inverse problems for particular types of partial differential equations, that is, for parabolic, hyperbolic and elliptic equations were studied quite well, see works [19-25] and the references therein. For instance, in $[22,25]$, there were studied inverse problems for heat equation in finding the time dependent right hand side; this was done by means of integral equations method.

In works by A.I. Prilepko and his pupils [26-28] there were considered inverse problems on determining the right hand side for particular types of partial differential equations. In works by V.V. Solov'ev [29,30], inverse problems on determining the right hand side $F(x, t)=$ $h(x, t) f(t)+g(x, t)$ were considered for a parabolic equation, where the unknown was the function $f(t)$. Work [31] by A.B. Kostin was devoted for studying the inverse problem for a parabolic equation on recovering a source, a right hand side $F(x, t)=h(x, t) f(x)$, where the unknown was the function $f(x)$. A.I. Kozhanov and R.R. Safiullova [32,33] studied inverse problem on determining both a solution to a parabolic equation and an unknown external source (right hand side).

In the present paper we first pose and study inverse Problems 2-4 on finding the factors in the right hand side of a mixed parabolic-hyperbolic equation with a degenerating hyperbolic part. The study is based on a solution to a direct initial boundary value Problem 1. As it was mentioned above, in works [11-15], a solution of this problem was constructed as a sum of orthogonal series and in justifying its convergence, the small denominators problem arisen. Because of this, we establish estimates on separation of the denominators from zero with an appropriate asymptotics and this allows us to justify the convergence of the series in the class of regular solutions of equation (1.1). On the base of the formula for solution to this problem, the resolving of inverse problems $2-4$ is equivalently reduced to solvability of loaded integral equations. Employing the theory of integral equations, we prove appropriate theorems on existence and uniqueness of solutions to the posed problem and provide explicit formulae for the solutions.

\section{Direct initial BOUNDARY VALUE PROBlem}

According [15], a solution of direct problem 11.2-1.5 is given by the series

$$
u(x, t)=\sqrt{\frac{2}{l}} \sum_{k=1}^{\infty} T_{k}(t) \sin \mu_{k} x, \quad \mu_{k}=\frac{\pi k}{l},
$$

where

$$
\begin{aligned}
& T_{k}(t)=\left\{\begin{array}{ll}
\frac{\omega_{k}(\alpha)}{\Delta_{k}(\alpha)} e^{-\lambda_{k}^{2} t}+f_{1 k} \int_{0}^{t} g_{1}(s) e^{-\lambda_{k}^{2}(t-s)} d s, & t>0, \\
\frac{\omega_{k}(\alpha)}{\Delta_{k}(\alpha)} \Delta_{k}(-t)-\omega_{k}(-t), & t<0,
\end{array}\right\} \\
& \Delta_{k}(-t)=\lambda_{k}^{2} \gamma_{\frac{1}{2 q}}(k) \sqrt{-t} J_{\frac{1}{2 q}}\left(p_{k}(-t)^{q}\right)+\gamma_{-\frac{1}{2 q}}(k) \sqrt{-t} J_{-\frac{1}{2 q}}\left(p_{k}(-t)^{q}\right), \\
& \gamma_{\frac{1}{2 q}}(k)=\frac{1}{2 q} \Gamma\left(\frac{1}{2 q}\right)\left(\frac{2}{p_{k}}\right)^{\frac{1}{2 q}}, \quad \gamma_{-\frac{1}{2 q}}(k)=-\frac{1}{2 q} \Gamma\left(-\frac{1}{2 q}\right)\left(\frac{2}{p_{k}}\right)^{-\frac{1}{2 q}}, \\
& f_{i k}=\sqrt{\frac{2}{l}} \int_{0}^{l} f_{i}(x) \sin \mu_{k} x d x, \quad i=1,2, \\
& \omega_{k}(-t)=f_{1 k} g_{1}(0) \gamma_{\frac{1}{2 q}}(k) \sqrt{-t} J_{\frac{1}{2 q}}\left(p_{k}(-t)^{q}\right)-f_{2 k} W_{k}(-t),
\end{aligned}
$$




$$
\begin{aligned}
& W_{k}(-t)=\frac{\pi}{2 q \sin \frac{\pi}{2 q}} \int_{t}^{0} g_{2}(s) \sqrt{s t} W(s,-t) d s \\
& W(s,-t)=J_{\frac{1}{2 q}}\left(p_{k}(-t)^{q}\right) J_{-\frac{1}{2 q}}\left(p_{k}(-s)^{q}\right)-J_{-\frac{1}{2 q}}\left(p_{k}(-t)^{q}\right) J_{\frac{1}{2 q}}\left(p_{k}(-s)^{q}\right),
\end{aligned}
$$

and $J_{\frac{1}{2 q}}(z)$ and $J_{-\frac{1}{2 q}}(z)$ are the Bessel function of first kind, $\Gamma(\cdot)$ is the Gamma function,

$$
\lambda_{k}^{2}=b+\mu_{k}^{2}, \quad p_{k}=\frac{\lambda_{k}}{q}, \quad q=\frac{m+2}{2}, \quad \tilde{\lambda}_{k}=\lambda_{k} l, \quad \alpha_{q l}=\frac{\alpha^{q}}{(q l)},
$$

under the assumption that

$$
\Delta_{k}(\alpha)=\lambda_{k}^{2} \gamma_{\frac{1}{2 q}}(k) \sqrt{\alpha} J_{\frac{1}{2 q}}\left(\widetilde{\lambda}_{k} \alpha_{q l}\right)+\gamma_{-\frac{1}{2 q}}(k) \sqrt{\alpha} J_{-\frac{1}{2 q}}\left(\widetilde{\lambda}_{k} \alpha_{q l}\right) \neq 0 .
$$

for all $k \in \mathbb{N}$.

In what follows we suppose that $b=\mu^{2} \geqslant 0, \mu \geqslant 0$, since if $b<0$, then starting with some index $k_{0}$, for all $k>k_{0}$, we have $b+\mu_{k}^{2}>0$, that is, the sign of $b$ makes no influence on the obtained results.

It was shown in works $[14,15]$ that $\Delta_{k}(\alpha)$ has countably many zeroes. This set coincides with the zero set of a linear combination of the Bessel functions $J_{\frac{1}{2 q}}\left(\widetilde{\lambda}_{k} \alpha_{q l}\right)$ and $J_{-\frac{1}{2 q}}\left(\widetilde{\lambda}_{k} \alpha_{q l}\right)$. Since $\Delta_{k}(\alpha)$ is involved into the denominators of the coefficients of series $(2.1)$, the small denominator problem arises $[34,14,15]$. This is why to justify the convergence of series (2.1), we need to show the existence of numbers $\alpha, l, m$ and $b$, under which the expression $\Delta_{k}(\alpha)$ is separated from the zero.

Lemma 2.1. If $\alpha_{q l}=p / t$ is an arbitrary fractional number, where $p$ and $t$ are coprime natural numbers and $\frac{r}{t} \neq \frac{3 q+1}{4 q}$ as $r=\overline{1, t-1}$, then there exist positive constants $k_{0} \in \mathbb{N}$ and $C_{0}$ such that for all $k>k_{0}$ and fixed $\mu \geqslant 0$ the estimate

$$
\left|k^{-1-\lambda} \Delta_{k}(\alpha)\right| \geqslant C_{0}>0, \quad \lambda=\frac{1}{2}-\frac{1}{2 q} .
$$

holds true.

The proof was given in work [15].

On the base of this lemma, we establish the following statement.

Theorem 2.1. Let the assumptions of Lemma 2.1 hold, $g_{1}(t) \in C[0, \beta], g_{2}(t) \in C[-\alpha, 0]$, $f_{i}(x) \in C^{3}[0, l], f_{i}(0)=f_{i}(l)=f_{i}^{\prime \prime}(0)=f_{i}^{\prime \prime}(l)=0, i=1,2$. If $\Delta_{k}(\alpha) \neq 0$ as $k=\overline{1, k_{0}}$, then there exists the unique solution of problem (1.2)-(1.5) determined by series (2.1) with coefficients given by formula (2.2).

The proof is similar to work [15].

\section{InVERSE Problem 2}

On the base of the formula for solution of direct problem studied in Section 2, we consider Inverse Problem 2. Supposing that function (2.1) satisfies condition (1.7), we get

$$
\sqrt{\frac{2}{l}} \sum_{k=1}^{\infty}\left[\frac{\omega_{k}(\alpha)}{\Delta_{k}(\alpha)} e^{-\lambda_{k}^{2} t}+f_{1 k} \int_{0}^{t} g_{1}(s) e^{-\lambda_{k}^{2}(t-s)} d s\right] \sin \mu_{k} x_{0}=h_{1}(t), \quad 0 \leqslant t \leqslant \beta,
$$

or

$$
g_{1}(0) \sqrt{\frac{2}{l}} \sum_{k=1}^{\infty} \frac{f_{1 k} \gamma_{\frac{1}{2 q}}(k) \sqrt{\alpha} J_{\frac{1}{2 q}}\left(p_{k} \alpha^{q}\right)}{\Delta_{k}(\alpha)} e^{-\lambda_{k}^{2} t} \sin \mu_{k} x_{0}-\sqrt{\frac{2}{l}} \sum_{k=1}^{\infty} \frac{f_{2 k} W_{k}(\alpha)}{\Delta_{k}(\alpha)} e^{-\lambda_{k}^{2} t} \sin \mu_{k} x_{0}
$$




$$
+\sqrt{\frac{2}{l}} \sum_{k=1}^{\infty} f_{1 k} \int_{0}^{t} g_{1}(s) e^{-\lambda_{k}^{2}(t-s)} d s \sin \mu_{k} x_{0}=h_{1}(t) .
$$

In the latter term we interchange the integration and summation that is possible by the uniform convergence of series 2.1) on $\bar{D}[15]$. Then for the function $g_{1}(t)$ we obtain a loaded first kind Volterra equation:

$$
\int_{0}^{t} g_{1}(s) K_{1}(s, t) d s=\widetilde{h}_{1}(t), \quad 0 \leqslant t \leqslant \beta
$$

with the kernel

$$
K_{1}(s, t)=\sqrt{\frac{2}{l}} \sum_{k=1}^{\infty} f_{1 k} e^{-\lambda_{k}^{2}(t-s)} \sin \mu_{k} x_{0}
$$

and the right hand side

$$
\widetilde{h}_{1}(t)=h_{1}(t)-g_{1}(0) H_{1}(t)+H_{2}(t)
$$

Here

$$
\begin{aligned}
& H_{1}(t)=\sqrt{\frac{2}{l}} \sum_{k=1}^{\infty} \frac{f_{1 k} e^{-\lambda_{k}^{2} t}}{\Delta_{k}(\alpha)} \gamma_{\frac{1}{2 q}}(k) \sqrt{\alpha} J_{\frac{1}{2 q}}\left(p_{k} \alpha^{q}\right) \sin \mu_{k} x_{0}, \\
& H_{2}(t)=\sqrt{\frac{2}{l}} \sum_{k=1}^{\infty} \frac{f_{2 k} e^{-\lambda_{k}^{2} t}}{\Delta_{k}(\alpha)} W_{k}(\alpha) \sin \mu_{k} x_{0} .
\end{aligned}
$$

Lemma 3.1. If the functions $f_{i}(x), i=1,2$, obey the assumptions of Theorem 2.1, then series (3.2), (3.4) and (3.5) and their derivatives in $t$ converge uniformly on a closed set $0 \leqslant s \leqslant t \leqslant \beta$.

Proof. As $0 \leqslant s \leqslant t \leqslant \beta$, series $(3.2)$ and its derivative in $t$ are majorized respectively by the series

$$
M_{1} \sum_{k=1}^{\infty}\left|f_{1 k}\right| \quad \text { and } \quad M_{2} \sum_{k=1}^{\infty} k^{2}\left|f_{1 k}\right|
$$

Hereinafter $M_{i}$ are positive constants.

Thanks to Lemma 2.1, Lemma 2.5 in [15] and asymptotic estimate [35]

$$
J_{\nu}(z)=\sqrt{\frac{2}{\pi z}} \cos \left(z-\frac{\pi}{2} \nu-\frac{\pi}{4}\right)+O\left(z^{-5 / 2}\right), \quad z \rightarrow \infty
$$

we can estimate the expressions in the sum in 3.4 and 3.5 for large $k$ :

$$
\begin{aligned}
& \frac{\left|f_{1 k} e^{-\lambda_{k}^{2} t}\right|\left|\gamma_{\frac{1}{2 q}}(k)\right| \sqrt{\alpha}\left|J_{\frac{1}{2 q}}\left(p_{k} \alpha^{q}\right)\right|}{\left|\Delta_{k}(\alpha)\right|} \leqslant \frac{M_{3}\left|f_{1 k}\right| k^{-1+\lambda}}{k^{1+\lambda} C_{0}} \leqslant M_{4} k^{-2}\left|f_{1 k}\right|, \\
& \frac{\left|f_{2 k} e^{-\lambda_{k}^{2} t}\right|\left|W_{k}(\alpha)\right|}{\left|\Delta_{k}(\alpha)\right|} \leqslant \frac{M_{5}\left|f_{2 k}\right| k^{-1+\lambda}}{k^{1+\lambda} C_{0}} \leqslant M_{6} k^{-2}\left|f_{2 k}\right| .
\end{aligned}
$$

For the expressions in the sum in the derivatives $H_{1}^{\prime}(t)$ and $H_{2}^{\prime}(t)$, we obtain the following estimates:

$$
\frac{\left|f_{1 k} \lambda_{k}^{2} e^{-\lambda_{k}^{2} t}\right|\left|\gamma_{\frac{1}{2 q}}(k)\right| \sqrt{\alpha}\left|J_{\frac{1}{2 q}}\left(p_{k} \alpha^{q}\right)\right|}{\left|\Delta_{k}(\alpha)\right|} \leqslant M_{7}\left|f_{1 k}\right|, \quad \frac{\left|f_{1 k} \lambda_{k}^{2} e^{-\lambda_{k}^{2} t}\right|\left|W_{k}(\alpha)\right|}{\left|\Delta_{k}(\alpha)\right|} \leqslant M_{8}\left|f_{2 k}\right| .
$$


This implies that on the closed set $0 \leqslant s \leqslant t \leqslant \beta$, series (3.2), (3.4) and (3.5) and their first derivatives are majorized respectively by the series

$$
M_{9} \sum_{k=1}^{\infty}\left|f_{1 k}\right|, \quad M_{10} \sum_{k=1}^{\infty} k^{2}\left|f_{2 k}\right| .
$$

Then under the made assumptions series (3.7) converge. This is why series $(3.2),(3.4),(3.5)$ and the series obtained by termwise differentiation in $t$ converge uniformly an $0 \leqslant s \leqslant t \leqslant \beta$.

Differentiating equation (3.1) in $t$, we have

$$
K_{1}(t, t) g_{1}(t)+\int_{0}^{t} g_{1}(s) \frac{\partial K_{1}(s, t)}{\partial t} d s=\widetilde{h}_{1}^{\prime}(t) .
$$

Letting $s=t$ in $(3.2)$, we get

$$
K_{1}(t, t)=\sum_{k=1}^{+\infty} f_{1 k} \sin \mu_{k} x_{0}=f_{1}\left(x_{0}\right) .
$$

The right hand in identity (3.9) is the expansion of the function $f_{1}(x)$ into the series $\left\{\sqrt{\frac{2}{l}} \sin \mu_{k} x\right\}_{k \geqslant 1}$ at the point $x=x_{0}$. If $f_{1}\left(x_{0}\right) \neq 0$, then by equation 3.8 we get the integral equation

$$
g_{1}(t)-\lambda \int_{0}^{t} g_{1}(s) \frac{\partial K_{1}(s, t)}{\partial t} d s=\frac{\widetilde{h}_{1}^{\prime}(t)}{f_{1}\left(x_{0}\right)}
$$

where

$$
\lambda=-\frac{1}{f_{1}\left(x_{0}\right)} .
$$

Equation (3.10) is an integral second kind Volterra equation with a continuous kernel and a continuous right hand side. As it is known, see, for instance, [37], such equation possesses the unique solution in the class $C[0, \beta]$ for each $g_{1}(0)$. Let us find this constant involved in the right hand side of equation (3.10). In order to do this, we let $t=0$ in equation (3.10) and we obtain

This implies

$$
f_{1}\left(x_{0}\right) g_{1}(0)=h_{1}^{\prime}(0)-g_{1}(0) H_{1}^{\prime}(0)+H_{2}^{\prime}(0)
$$

under the condition

$$
g_{1}(0)=\frac{h_{1}^{\prime}(0)+H_{2}^{\prime}(0)}{f_{1}\left(x_{0}\right)+H_{1}^{\prime}(0)}
$$

$$
\begin{aligned}
f_{1}\left(x_{0}\right)+H_{1}^{\prime}(0) & =\sqrt{\frac{2}{l}} \sum_{k=1}^{+\infty} f_{1 k} \sin \mu_{k} x_{0}-\sqrt{\frac{2}{l}} \sum_{k=1}^{+\infty} \frac{f_{1 k} \lambda_{k}^{2} \gamma_{\frac{1}{2 q}}(k) \sqrt{\alpha} J_{\frac{1}{2 q}}\left(p_{k} \alpha^{q}\right)}{\Delta_{k}(\alpha)} \sin \mu_{k} x_{0} \\
& =\sqrt{\frac{2}{l}} \sum_{k=1}^{+\infty} f_{1 k}\left[1-\frac{\left.\lambda_{k}^{2} \gamma_{\frac{1}{2 q}}(k) \sqrt{\alpha} J_{\frac{1}{2 q}}\left(p_{k} \alpha^{q}\right)\right]}{\Delta_{k}(\alpha)}\right] \sin \mu_{k} x_{0} \\
& =\sqrt{\frac{2}{l}} \sum_{k=1}^{+\infty} \frac{f_{1 k} \gamma_{-\frac{1}{2 q}}(k) \sqrt{\alpha} J_{-\frac{1}{2 q}}\left(p_{k} \alpha^{q}\right)}{\Delta_{k}(\alpha)} \sin \mu_{k} x_{0} \neq 0 .
\end{aligned}
$$

For instance, let $q=1$ and $\mu=0$. Then condition 3.12 becomes

$$
\sqrt{\frac{2}{l}} \sum_{k=1}^{+\infty} \frac{f_{1 k} \cos \lambda_{k} \alpha}{\lambda_{k} \sin \lambda_{k} \alpha+\cos \lambda_{k} \alpha} \sin \mu_{k} x_{0} \neq 0
$$


and this holds as $\alpha_{l}=\alpha / l=p \in \mathbb{N}$.

Equation (3.8) with $g_{1}(0)$ defined by formula (3.11) is a classical second kind Volterra equation, whose solution is constructed easily by the successive approximations method.

Thus, we have proved the following theorem.

Theorem 3.1. Suppose that the assumptions of Lemma 2.1 hold true, the functions $f_{i}(x)$, $i=1,2$, and $g_{2}(t)$ satisfy the assumptions of Theorem 2.1, $h_{1}(t) \in C^{1}[0, \beta], f_{1}\left(x_{0}\right) \neq 0$. If condition (3.12) is satisfied, then integral equation (3.8) has a unique solution $g_{1}(t) \in C[0, \beta]$ and Problem 2 also has a unique solution. If condition (3.12) fails, integral equation (3.8) and Problem 2 has solution unique to a term, whose factor is an unknown number $g_{1}(0)$.

Now we are going to show that condition $f_{1}\left(x_{0}\right) \neq 0$ is essential for the unique solvability of Problem 2. Indeed, there exists a function $f_{1}(x)=\sin \mu_{m} x=\sin \pi m \widetilde{x}$, where $m$ is some fixed natural number and $\widetilde{x}=x / l$ such that $f_{1}\left(x_{0}\right)=\sin \pi m \widetilde{x}_{0}=0$. For such function, arbitrary $g_{1}(t) \in C[0, \beta]$ and $h_{1}(t) \equiv 0$, there exists a nonzero solution to Problem 2

$$
u_{1 m}(x, t)=T_{1 m}(t) \sin \mu_{m} x
$$

where

$$
\begin{aligned}
& T_{1 m}(t)= \begin{cases}\frac{\omega_{1 m}(\alpha)}{\Delta_{m}(\alpha)} e^{-\lambda_{m}^{2} t}+\int_{0}^{t} g_{1}(s) e^{-\lambda_{m}^{2}(t-s)} d s, & t>0, \\
\frac{\omega_{1 m}(\alpha)}{\Delta_{m}(\alpha)} \Delta_{m}(-t)-\omega_{1 m}(-t), & t<0,\end{cases} \\
& \omega_{1 m}(-t)=g_{1}(0) \gamma_{\frac{1}{2 q}}(m) \sqrt{-t} J_{\frac{1}{2 q}}\left(p_{m}(-t)^{q}\right)-f_{2 m} W_{m}(-t) .
\end{aligned}
$$

Indeed, function (3.13) satisfies conditions $(1.2)-(1.7)$ with $h_{1}(t) \equiv 0$. The belonging to the class 1.2 is because thanks to the asymptotic formula [35]

$$
J_{\nu}(z) \sim \frac{1}{\Gamma(1+\nu)}\left(\frac{z}{2}\right)^{\nu} \quad \text { as } \quad z \rightarrow 0
$$

and Lemma 2.1 we have

$$
\begin{aligned}
\lim _{t \rightarrow 0+0} u_{1 m}(x, t)= & \frac{\omega_{1 m}(\alpha)}{\Delta_{m}(\alpha)} \sin \mu_{m} x=\lim _{t \rightarrow 0-0} u_{1 m}(x, t) \\
= & \lim _{t \rightarrow 0-0}\left[\frac{\omega_{1 m}(\alpha)}{\Delta_{m}(\alpha)} \Delta_{m}(-t)-\omega_{1 m}(-t)\right] \sin \mu_{m} x \\
= & \sin \mu_{m} x \lim _{t \rightarrow 0-0} \frac{\omega_{1 m}(\alpha)}{\Delta_{m}(\alpha)}\left[\lambda_{m}^{2} \sqrt{-t} \gamma_{\frac{1}{2 q}}(s) J_{\frac{1}{2 q}}\left(p_{m}(-t)^{q}\right)+\right. \\
& \left.+\sqrt{-t} \gamma_{-\frac{1}{2 q}}(m) J_{-\frac{1}{2 q}}\left(p_{m}(-t)^{q}\right)\right]- \\
& -\sin \mu_{m} x \lim _{t \rightarrow 0-0}\left[g_{1}(0) \gamma_{\frac{1}{2 q}}(m) \sqrt{-t} J_{\frac{1}{2 q}}\left(p_{m}(-t)^{q}\right)-f_{2 m} W_{m}(-t)\right] \\
= & \sin \mu_{m} x \lim _{t \rightarrow 0-0}\left[\frac{\omega_{1 m}(\alpha)}{\Delta_{m}(\alpha)}\left(-\lambda_{m}^{2} t+1\right)+g_{1}(0) t-f_{2 m} W_{m}(-t)\right]=\frac{\omega_{1 m}(\alpha)}{\Delta_{m}(\alpha)} \sin \mu_{m} x .
\end{aligned}
$$

Thanks to the differentiability of the asymptotic estimate for the Bessel function, it is easy to show that the normal derivatives at the type changing line:

$$
\lim _{t \rightarrow 0+0} \frac{\partial u_{1 m}(x, t)}{\partial t}=\left[-\frac{\omega_{1 m}(\alpha)}{\Delta_{m}(\alpha)} \lambda_{m}^{2}+g_{1}(0)\right] \sin \mu_{m} x=\lim _{t \rightarrow 0-0} \frac{\partial u_{m}(x, t)}{\partial t} .
$$


Conditions (1.4) and (1.5) are also satisfied since

$$
\begin{aligned}
& u_{1 m}(x,-\alpha)=\left[\frac{\omega_{1 m}(\alpha)}{\Delta_{m}(\alpha)} \Delta_{m}(\alpha)-\omega_{1 m}(\alpha)\right] \sin \mu_{m} x=0, \\
& u_{1 m}\left(x_{0}, t\right)=T_{1 m}(t) \sin \mu_{m} x_{0}=0 .
\end{aligned}
$$

It follows from the construction of function $(3.13)$ that on the set $D_{-} \cup D_{+}$, the function solves equation (1.1).

Let us find out for which points $\widetilde{x}_{0}$ in $(0,1)$ the identity

$$
\sin \pi m \widetilde{x}_{0}=0 \quad \Longleftrightarrow \quad \widetilde{x}_{0}=\frac{k}{m}, \quad k, m \in \mathbb{N}, \quad k<m,
$$

holds. We see that as $\widetilde{x}_{0}$ is irrational, the condition $f_{1}\left(x_{0}\right) \neq 0$ fails.

\section{Problem 3}

Here we consider Problem 3 on finding a factor in the time-dependent right hand side in equation (1.1). Similar to Section 3, letting $x=x_{0}$ in formula (2.1) and supposing that a function satisfies condition (1.9), we obtain:

$$
\begin{aligned}
& \sqrt{\frac{2}{l}} \sum_{k=1}^{\infty}\left[\frac{\omega_{k}(\alpha)}{\Delta_{k}(\alpha)} \Delta_{k}(-t) \sin \mu_{k} x_{0}-\omega_{k}(-t)\right] \sin \mu_{k} x_{0} \\
& =\sqrt{\frac{2}{l}} \sum_{k=1}^{\infty} \frac{f_{1 k} g_{1}(0) \gamma_{\frac{1}{2 q}}(k) \sqrt{\alpha} J_{\frac{1}{2 q}}\left(p_{k} \alpha^{q}\right)-f_{2 k} W_{k}(\alpha)}{\Delta_{k}(\alpha)} \Delta_{k}(-t) \sin \mu_{k} x_{0} \\
& -\sqrt{\frac{2}{l}} \sum_{k=1}^{\infty}\left[f_{1 k} g_{1}(0) \gamma_{\frac{1}{2 q}}(k) \sqrt{-t} J_{\frac{1}{2 q}}\left(p_{k}(-t)^{q}\right)-f_{2 k} W_{k}(-t)\right] \sin \mu_{k} x_{0}=h_{2}(t)
\end{aligned}
$$

or

$$
\int_{t}^{0} g_{2}(s) K_{2}(s, t) d s-\int_{-\alpha}^{0} g_{2}(s) K_{3}(s, t) d s=\widetilde{h}_{2}(t), \quad-\alpha \leqslant t \leqslant 0 .
$$

Here

$$
\begin{aligned}
& K_{2}(s, t)=\sqrt{\frac{2}{l}} \frac{\pi}{2 q \sin \frac{\pi}{2 q}} \sum_{k=1}^{\infty} f_{2 k} \sqrt{s t} W(s,-t) \sin \mu_{k} x_{0}, \quad-\alpha \leqslant t \leqslant s \leqslant 0, \\
& K_{3}(s, t)=\sqrt{\frac{2}{l}} \frac{\pi}{2 q \sin \frac{\pi}{2 q}} \sum_{k=1}^{\infty} \frac{f_{2 k}}{\Delta_{k}(\alpha)} \Delta_{k}(-t) \sqrt{-s \alpha} W(s, \alpha) \sin \mu_{k} x_{0}, \\
& \widetilde{h}_{2}(t)=h_{2}(t)-g_{1}(0) H_{3}(t), \quad-\alpha \leqslant t \leqslant 0, \\
& H_{3}(t)=\sqrt{\frac{2}{l}} \sum_{k=1}^{\infty} f_{1 k}\left[\frac{\gamma_{\frac{1}{2 q}}(k) \sqrt{\alpha} J_{\frac{1}{2 q}}\left(p_{k} \alpha^{q}\right)}{\Delta_{k}(\alpha)} \Delta_{k}(-t)-\gamma_{\frac{1}{2 q}}(k) \sqrt{-t} J_{\frac{1}{2 q}}\left(p_{k}(-t)^{q}\right)\right] \sin \mu_{k} x_{0} .
\end{aligned}
$$

Lemma 4.1. Let the functions $f_{i}(x), i=1,2$, satisfy the assumptions of Theorem 2.1, then series (4.2), (4.3) and (4.5) and their derivatives in t up to the second order converge uniformly on the set $-\alpha \leqslant t \leqslant s \leqslant 0$.

Proof. By Lemma 2.1 and asymptotic estimate (3.6), we estimates the expressions in the sum in (4.2), 4.3) and (4.5). The expression $W(s,-t)$ in (2.3) and its derivatives in $t$ up to the second order are estimated as follows:

$$
\begin{aligned}
|\sqrt{s t} W(s,-t)| & \leqslant\left|\sqrt{s t} J_{\frac{1}{2 q}}\left(p_{k}(-t)^{q}\right) J_{-\frac{1}{2 q}}\left(p_{k}(-s)^{q}\right)\right|+\left|\sqrt{s t} J_{-\frac{1}{2 q}}\left(p_{k}(-t)^{q}\right) J_{\frac{1}{2 q}}\left(p_{k}(-s)^{q}\right)\right| \\
& \leqslant M_{11} k^{-\frac{1}{2}-\frac{1}{2}}+M_{12} k^{-\frac{1}{2}-\frac{1}{2}} \leqslant M_{13} k^{-1}
\end{aligned}
$$




$$
\begin{aligned}
\left|[\sqrt{s t} W(s,-t)]_{t}^{\prime}\right| \leqslant & \lambda_{k}\left|\sqrt{-s}(-t)^{q-\frac{1}{2}} J_{\frac{1}{2 q}-1}\left(p_{k}(-t)^{q}\right) J_{-\frac{1}{2 q}}\left(p_{k}(-s)^{q}\right)\right| \\
& +\lambda_{k}\left|\sqrt{-s}(-t)^{q-\frac{1}{2}} J_{1-\frac{1}{2 q}}\left(p_{k}(-t)^{q}\right) J_{\frac{1}{2 q}}\left(p_{k}(-s)^{q}\right)\right| \\
\leqslant & M_{14} k^{1-\frac{1}{2}-\frac{1}{2}}+M_{15} k^{1-\frac{1}{2}-\frac{1}{2}} \leqslant M_{16} .
\end{aligned}
$$

Since $W_{t}^{\prime \prime}(s,-t)=-\lambda_{k}^{2}(-t)^{m} W(s,-t)$, thanks to estimate 4.6 we get

$$
\left|[\sqrt{s t} W(s,-t)]_{t t}^{\prime \prime}\right| \leqslant M_{17} k \text {. }
$$

Similar to [15], we estimate the expression $\Delta_{k}(t)$ in $(2.2)$ and its derivatives up to the second order:

$$
\begin{aligned}
\left|\Delta_{k}(-t)\right| & \leqslant \lambda_{k}^{2}\left|\gamma_{\frac{1}{2 q}}(k) \sqrt{-t} J_{\frac{1}{2 q}}\left(p_{k}(-t)^{q}\right)\right|+\left|\gamma_{-\frac{1}{2 q}}(k) \sqrt{-t} J_{-\frac{1}{2 q}}\left(p_{k}(-t)^{q}\right)\right| \\
& \leqslant M_{18} k^{2-\frac{1}{2 q}-\frac{1}{2}}+M_{19} k^{\frac{1}{2 q}-\frac{1}{2}} \leqslant M_{20} k^{1+\lambda} \\
\left|\Delta_{k}^{\prime}(-t)\right| & \leqslant \lambda_{k}^{3}\left|\gamma_{\frac{1}{2 q}}(k) \sqrt{-t} J_{\frac{1}{2 q}-1}\left(p_{k}(-t)^{q}\right)\right|+\lambda_{k}\left|\gamma_{-\frac{1}{2 q}}(k) \sqrt{-t} J_{1-\frac{1}{2 q}}\left(p_{k}(-t)^{q}\right)\right| \\
& \leqslant M_{21} k^{3-\frac{1}{2 q}-\frac{1}{2}}+M_{22} k^{1+\frac{1}{2 q}-\frac{1}{2}} \leqslant M_{23} k^{2+\lambda} .
\end{aligned}
$$

Since $\Delta_{k}^{\prime \prime}(-t)=-\lambda_{k}^{2}(-t)^{m} \Delta_{k}(-t)$, by estimate 4.9 we obtain

$$
\left|\Delta_{k}^{\prime \prime}(-t)\right| \leqslant M_{24} k^{3+\lambda}
$$

In view of obtained estimates 4.6-4.11), for expression in the sum 4.3) we obtain the following estimates

$$
\begin{aligned}
& \left|\frac{f_{2 k}}{\Delta_{k}(\alpha)} \Delta_{k}(-t) \sqrt{-s \alpha} W(s, \alpha)\right| \leqslant M_{25}\left|f_{2 k}\right| \frac{k^{1+\lambda-1}}{k^{1+\lambda}} \leqslant M_{26}\left|f_{2 k}\right| k^{-1}, \\
& \left|\frac{f_{2 k}}{\Delta_{k}(\alpha)} \Delta_{k}^{\prime}(-t) \sqrt{-s \alpha} W(s, \alpha)\right| \leqslant M_{27}\left|f_{2 k}\right| \frac{k^{2+\lambda-1}}{k^{1+\lambda}} \leqslant M_{28}\left|f_{2 k}\right|, \\
& \left|\frac{f_{2 k}}{\Delta_{k}(\alpha)} \Delta_{k}^{\prime \prime}(-t) \sqrt{-s \alpha} W(s, \alpha)\right| \leqslant M_{29}\left|f_{2 k}\right| \frac{k^{3+\lambda-1}}{k^{1+\lambda}} \leqslant M_{30}\left|f_{2 k}\right| k .
\end{aligned}
$$

In the same way, estimating the expression in the sum in (4.5), we obtain

$$
\begin{aligned}
& \left|f_{1 k}\right|\left|\frac{\gamma_{\frac{1}{2 q}}(k) \sqrt{\alpha} J_{\frac{1}{2 q}}\left(p_{k} \alpha^{q}\right)}{\Delta_{k}(\alpha)} \Delta_{k}(-t)\right|+\left|f_{1 k}\right|\left|\gamma_{\frac{1}{2 q}}(k) \sqrt{-t} J_{\frac{1}{2 q}}\left(p_{k}(-t)^{q}\right)\right| \\
& \quad \leqslant M_{31}\left|f_{1 k}\right| \frac{k^{-1+\lambda}}{k^{1+\lambda}} k^{1+\lambda}+M_{32}\left|f_{1 k}\right| k^{-1+\lambda} \leqslant M_{33}\left|f_{1 k}\right| k^{-1+\lambda}, \\
& \left|f_{1 k}\right|\left|\frac{\gamma_{\frac{1}{2 q}}(k) \sqrt{\alpha} J_{\frac{1}{2 q}}\left(p_{k} \alpha^{q}\right)}{\Delta_{k}(\alpha)} \Delta_{k}^{\prime}(-t)\right|+\left|f_{1 k}\right| \lambda_{k}\left|\gamma_{\frac{1}{2 q}}(k)(-t)^{q-\frac{1}{2}} J_{\frac{1}{2 q}-1}\left(p_{k}(-t)^{q}\right)\right| \\
& \leqslant M_{34}\left|f_{1 k}\right| \frac{k^{-1+\lambda}}{k^{1+\lambda}} k^{2+\lambda}+M_{35}\left|f_{1 k}\right| k^{1-1+\lambda} \leqslant M_{36}\left|f_{1 k}\right| k^{\lambda}, \\
& \left|f_{1 k}\right|\left|\frac{\gamma_{\frac{1}{2 q}}(k) \sqrt{\alpha} J_{\frac{1}{2 q}}\left(p_{k} \alpha^{q}\right)}{\Delta_{k}(\alpha)} \Delta_{k}^{\prime \prime}(-t)\right|+\left|f_{1 k}\right| \lambda_{k}^{2}(-t)^{m}\left|\gamma_{\frac{1}{2 q}}(k) \sqrt{-t} J_{\frac{1}{2 q}}\left(p_{k}(-t)^{q}\right)\right| \\
& \leqslant M_{37}\left|f_{1 k}\right| \frac{k^{-1+\lambda}}{k^{1+\lambda}} k^{3+\lambda}+M_{38}\left|f_{1 k}\right| k^{2-1+\lambda} \leqslant M_{39}\left|f_{1 k}\right| k^{1+\lambda} .
\end{aligned}
$$


This yields that on the closed set $-\alpha \leqslant t \leqslant s \leqslant 0$, series (4.2), (4.3) and (4.5) and their derivatives in up to the second order are majorized respectively by the series

$$
M_{40} \sum_{k=1}^{\infty} k^{1+\lambda}\left|f_{1 k}\right|, \quad M_{42} \sum_{k=1}^{\infty} k\left|f_{2 k}\right| .
$$

Under the made assumptions, series 4.18 converge. Then series 4.2 , 4.3), 4.5 and the series obtained by twise termwise differentiation in $t$ converge uniformly on $-\alpha \leqslant t \leqslant s \leqslant 0$.

We differentiate equation (4.1) in $t$ and we obtain:

$$
-g_{2}(t) K_{2}(t, t)+\int_{t}^{0} g_{2}(s) \frac{\partial K_{2}(s, t)}{\partial t} d s-\int_{-\alpha}^{0} g_{2}(s) \frac{\partial K_{3}(s, t)}{\partial t} d s=\widetilde{h}_{2}^{\prime}(t) .
$$

By identity we see 4.2 that

$$
\left.K_{2}(s, t)\right|_{s=t}=0 \quad \text { since }\left.\quad W(s,-t)\right|_{s=t}=0 .
$$

Then differentiating equation 4.19 once again, we get

$$
-\left.g_{2}(t) \frac{\partial K_{2}(s, t)}{\partial t}\right|_{s=t}+\int_{t}^{0} g_{2}(s) \frac{\partial^{2} K_{2}(s, t)}{\partial t^{2}} d s-\int_{-\alpha}^{0} g_{2}(s) \frac{\partial^{2} K_{3}(s, t)}{\partial t^{2}} d s=\widetilde{h}_{2}^{\prime \prime}(t) .
$$

By 4.2. we calculate

$$
\begin{aligned}
\frac{\partial K_{2}(s, t)}{\partial t}= & \sqrt{\frac{2}{l}} \frac{\pi}{2 q \sin \frac{\pi}{2 q}} \sum_{k=1}^{\infty} f_{2 k} \sqrt{-s}[\sqrt{-t} W(s,-t)]_{t}^{\prime} \sin \mu_{k} x_{0} \\
= & \sqrt{\frac{2}{l}} \frac{\pi}{2 q \sin \frac{\pi}{2 q}} \sum_{k=1}^{\infty} f_{2 k} \sqrt{-s}\left[-\lambda_{k}(-t)^{q-1 / 2} J_{\frac{1}{2 q}-1}\left(p_{k}(-t)^{q}\right) J_{-\frac{1}{2 q}}\left(p_{k}(-s)^{q}\right)\right. \\
& \left.-\lambda_{k}(-t)^{q-1 / 2} J_{1-\frac{1}{2 q}}\left(p_{k}(-t)^{q}\right) J_{\frac{1}{2 q}}\left(p_{k}(-s)^{q}\right)\right] \sin \mu_{k} x_{0}
\end{aligned}
$$

Employing the identity [35]

we find

$$
J_{\nu}(z) J_{1-\nu}(z)+J_{-\nu}(z) J_{\nu-1}(z)=\frac{2}{\pi z} \sin \nu \pi
$$

$$
\left.\frac{\partial K_{2}(s, t)}{\partial t}\right|_{s=t}=-\sqrt{\frac{2}{l}} \sum_{k=1}^{+\infty} f_{2 k} \sin \mu_{k} x_{0}=-f_{2}\left(x_{0}\right) .
$$

If we suppse $f_{2}\left(x_{0}\right) \neq 0$, then by 4.20 and 4.21 we obtain a second kind Fredholm integral equation

$$
g_{2}(t)-\lambda \int_{-\alpha}^{0} g_{2}(s) H(s, t) d t=\mu(t)
$$

where

$$
\begin{aligned}
& H(s, t)=\left\{\begin{array}{cr}
\frac{\partial^{2} K_{3}(s, t)}{\partial t^{2}}, & -\alpha \leqslant s \leqslant t, \\
\frac{\partial^{2} K_{3}(s, t)}{\partial t^{2}}-\frac{\partial^{2} K_{2}(s, t)}{\partial t^{2}}, & t \leqslant s \leqslant 0,
\end{array}\right. \\
& \lambda=\frac{1}{f_{2}\left(x_{0}\right)}, \quad \mu(t)=\frac{\widetilde{h}_{2}^{\prime \prime}(t)}{f_{2}\left(x_{0}\right)} .
\end{aligned}
$$


The kernel $H(s, t)$ of integral equation 4.22 defined by formula 4.23$)$ is continuous on a closed square $-\alpha \leqslant s, t \leqslant 0$. If $h_{2}(t) \in C^{2}[-\alpha, 0]$, then the right hand side $\mu(t)$ is also continuous on $[-\alpha, 0]$. Therefore, equation $(4.22)$ is a second kind Fredholm integral equation with a continuous kernel and continuous right hand side, to which the Fredholm theorems can be applied. This means the Fredholm property of Problem 2. Let us select cases, when equation (4.22) has the unique solution [36]. By the successive approximation methods we can prove the unique solvability of this equation in the class of continuous on $[-\alpha, 0]$ function as

$$
|\lambda|<\frac{1}{M \alpha}, \quad M=\max _{-\alpha \leqslant s, t \leqslant 0}|H(s, t)| .
$$

It also follows from the Fredholm theory that if $\lambda$ is not a characteristic number of the kernel $H(s, t)$, then integral equation $(4.22)$ has the unique continuous on $[-\alpha, 0]$ solution.

Thus, we have proved the following theorem.

Theorem 4.1. Let the assumptions of Lemma 2.1 be satisfied, the functions $f_{i}(x), i=1,2$, and $g_{1}(t)$ satisfy the assumptions of Theorem 2.1, $h_{2}(t) \in C^{2}[-\alpha, 0], f_{2}\left(x_{0}\right) \neq 0$. Then under one of the following conditions: a) $\left|f_{2}\left(x_{0}\right)\right|>M \alpha$; b) the number $f_{2}^{-1}\left(x_{0}\right)$ is not a characteristic number of the kernel $H(s, t)$, there exists a unique solution to Problem 3. At that, the function $g_{2}(t)$ is introduced as the solution of integral equation (4.22), and then the function $u(x, t)$ is determined by formula (2.1).

We also note that the condition $f_{2}\left(x_{0}\right) \neq 0$ is essential for the unique solvability of Problem 3 . Indeed, there exists a function $f_{2}(x)=\sin \mu_{n} x=\sin \pi n \widetilde{x}$, where $n$ is some fixed natural number, $\widetilde{x}=x / l$, such that $f_{2}\left(x_{0}\right)=\sin \pi n \widetilde{x}_{0}=0$. Then for such function, all $g_{2}(t) \in C[-\alpha, 0]$ and $h_{2}(t) \equiv 0$ we can construct a non-zero solution to Problem 3:

$$
u_{2 n}(x, t)=T_{2 n}(t) \sin \mu_{n} x,
$$

where

$$
\begin{aligned}
& T_{1 n}(t)= \begin{cases}\frac{\omega_{2 n}(\alpha)}{\Delta_{n}(\alpha)} e^{-\lambda_{n}^{2} t}+f_{1 n} \int_{0}^{t} g_{1}(s) e^{-\lambda_{n}^{2}(t-s)} d s, & t>0, \\
\frac{\omega_{2 n}(\alpha)}{\Delta_{n}(\alpha)} \Delta_{n}(-t)-\omega_{2 n}(-t), & t<0,\end{cases} \\
& \omega_{2 n}(-t)=f_{1 n} g_{1}(0) \gamma_{\frac{1}{2 q}}(n) \sqrt{-t} J_{\frac{1}{2 q}}\left(p_{n}(-t)^{q}\right)-W_{n}(-t) .
\end{aligned}
$$

Similar to function (3.13), we prove that function (4.24) satisfies the conditions of Problem 3 for $h_{2}(t)=0$ and arbitrary function $g_{2}(t) \in C[-\alpha, 0]$.

\section{Problem 4}

Supposing that function (2.1) satisfies conditions 1.7 and 1.9 , we obtain the system of integral equations with loaded terms:

$$
\begin{aligned}
& \int_{0}^{t} g_{1}(s) K_{1}(s, t) d s-\int_{-\alpha}^{0} g_{2}(s) \widetilde{K}_{1}(s, t) d s=h_{1}(t)-g_{1}(0) H_{1}(t)=\widetilde{\widetilde{h}}_{1}(t), \\
& \int_{t}^{0} g_{2}(s) K_{2}(s, t) d t-\int_{-\alpha}^{0} g_{2}(s) K_{3}(s, t) d s=h_{2}(t)-g_{1}(0) H_{3}(t)=\widetilde{h}_{2}(t),
\end{aligned}
$$


where $K_{1}(s, t), H_{1}(t), K_{2}(s, t), K_{3}(s, t)$ and $H_{3}(t)$ are introduced by formulae (3.2), (3.4), (4.2), 4.3 and 4.5 ,

$$
\widetilde{K}_{1}(s, t)=\sqrt{\frac{2}{l}} \frac{\pi}{2 q \sin \frac{\pi}{2 q}} \sum_{k=1}^{+\infty} \frac{f_{2 k} e^{-\lambda_{k}^{2} t}}{\Delta_{k}(\alpha)} \sqrt{-s \alpha} W(s, \alpha) \sin \mu_{k} x_{0} .
$$

Following Sections 3 and 4, we differentiate equation (5.1) once, and twice equation (5.2). As a result we have

$$
\begin{gathered}
g_{1}(t) f_{1}\left(x_{0}\right)-\int_{0}^{t} g_{1}(s) \frac{\partial K_{1}(s, t)}{\partial t} d s-\int_{-\alpha}^{0} g_{2}(s) \frac{\partial \widetilde{K}_{1}(s, t)}{\partial t} d s=\widetilde{\widetilde{h}}_{1}^{\prime}(t), \\
-g_{2}(t) f_{2}\left(x_{0}\right)+\int_{t}^{0} g_{2}(s) \frac{\partial^{2} K_{2}(s, t)}{\partial t^{2}} d s-\int_{-\alpha}^{0} g_{2}(s) \frac{\partial^{2} K_{3}(s, t)}{\partial t^{2}} d s=\widetilde{h}_{2}^{\prime \prime}(t) .
\end{gathered}
$$

Letting $t=0$ in identities $(5.4)$ and $(5.5)$, we obtain

$$
\begin{gathered}
g_{1}(0) f_{1}\left(x_{0}\right)-\left.\int_{-\alpha}^{0} g_{2}(s) \frac{\partial \widetilde{K}_{1}(s, t)}{\partial t}\right|_{t=0} d s=h_{1}^{\prime}(0)-g_{1}(0) H_{1}^{\prime}(0), \\
-g_{2}(0) f_{2}\left(x_{0}\right)-\left.\int_{-\alpha}^{0} g_{2}(s) \frac{\partial^{2} K_{3}(s, t)}{\partial t^{2}}\right|_{t=0} d s=h_{2}^{\prime \prime}(0)-g_{1}(0) H_{3}^{\prime \prime}(0) .
\end{gathered}
$$

Thanks to formulae (5.3), 4.3), (3.4) and 4.5), we calculate

$$
\begin{aligned}
& \left.\frac{\partial \widetilde{K}_{1}(s, t)}{\partial t}\right|_{t=0}=-\sqrt{\frac{2}{l}} \frac{\pi}{2 q \sin \frac{\pi}{2 q}} \sum_{k=1}^{+\infty} \frac{f_{2 k} \lambda_{k}^{2}}{\Delta_{k}(\alpha)} \sqrt{-s \alpha} W(s, \alpha) \sin \mu_{k} x_{0}, \\
& \left.\frac{\partial^{2} K_{3}(s, t)}{\partial t^{2}}\right|_{t=0}=-\left.\lambda_{k}^{2}(-t)^{m} K_{3}(s, t)\right|_{t=0}=0, \\
& H_{1}^{\prime}(0)=-\sqrt{\frac{2}{l}} \sum_{k=1}^{\infty} \frac{\lambda_{k}^{2} f_{1 k}}{\Delta_{k}(\alpha)} \gamma_{\frac{1}{2 q}}(k) \sqrt{\alpha} J_{\frac{1}{2 q}}\left(p_{k} \alpha^{q}\right) \sin \mu_{k} x_{0}, \\
& \left.H_{3}^{\prime \prime}(t)\right|_{t=0}=-\left.\lambda_{k}^{2}(-t)^{m} H_{3}^{\prime \prime}(t)\right|_{t=0}=0 .
\end{aligned}
$$

By equation (5.6) we find

$$
g_{1}(0)=\left(f_{1}\left(x_{0}\right)+H_{1}^{\prime}(0)\right)^{-1}\left[h_{1}^{\prime}(0)+\left.\int_{-\alpha}^{0} g_{2}(s) \frac{\partial \widetilde{K}_{1}(s, t)}{\partial t}\right|_{t=0} d s\right]
$$

under the condition $f_{1}\left(x_{0}\right)+H_{1}^{\prime}(0) \neq 0$ and we substitute this in the right hand side in equation (5.5). Then if $f_{2}\left(x_{0}\right) \neq 0$, by (5.5) we obtain the integral equation

$$
g_{2}(t)-\lambda \int_{-\alpha}^{0} g_{2}(s) \widetilde{H}(s, t) d t=\widetilde{\mu}(t)
$$

where

$$
\begin{aligned}
\widetilde{H}(s, t)= & \frac{\partial^{2} K_{3}(s, t)}{\partial t^{2}}-\left.\frac{H_{3}^{\prime \prime}(t)}{f_{1}\left(x_{0}\right)+H_{1}^{\prime}(0)} \frac{\partial \widetilde{K}_{1}(s, t)}{\partial t}\right|_{t=0}, \quad-\alpha \leqslant s \leqslant t \\
& \frac{\partial^{2} K_{3}(s, t)}{\partial t^{2}}-\left.\frac{H_{3}^{\prime \prime}(t)}{f_{1}\left(x_{0}\right)+H_{1}^{\prime}(0)} \frac{\partial \widetilde{K}_{1}(s, t)}{\partial t}\right|_{t=0}+\frac{\partial^{2} K_{2}(s, t)}{\partial t^{2}}, \quad t \leqslant s \leqslant 0,
\end{aligned}
$$




$$
\lambda=\frac{1}{f_{2}\left(x_{0}\right)}, \quad \widetilde{\mu}(t)=\frac{H_{3}^{\prime \prime}(t) h_{1}^{\prime}(0)}{f_{2}\left(x_{0}\right)\left(f_{1}\left(x_{0}\right)+H_{1}^{\prime}(0)\right)}-\frac{h_{2}^{\prime \prime}(t)}{f_{2}\left(x_{0}\right)} .
$$

Equation (5.8) is a second kind Fredholm integral equation with a continuous kernel and continuous right hand side, to which we can apply the Fredholm theory [36].

Once we find the function $g_{2}(s)$ by equation (5.8), we can find the function $g_{1}(s)$ by $(5.4)$ as a solution of a second kind Volterra integral equation with a continuous kernel and continuous right hand side under the condition $f_{1}\left(x_{0}\right) \neq 0$ and 3.12 .

Thus, we arrive at the following statement.

Theorem 5.1. Let the assumptions of Lemma 2.1 hold, the functions $f_{i}(x), i=1,2$, satisfy the assumptions of Theorem 2.1, $h_{1}(t) \in C^{1}[0, \beta], h_{2}(t) \in C^{2}[-\alpha, 0], f_{1}\left(x_{0}\right) \neq 0, f_{2}\left(x_{0}\right) \neq 0$, inequality (3.12) hold as well as one of the following conditions: a) $\left|f_{2}\left(x_{0}\right)\right|>M \alpha$, where $M=\max _{-\alpha \leqslant s, t \leqslant 0}|H(s, t)|$; b) the number $f_{2}^{-1}\left(x_{0}\right)$ is not a characteristic number of the kernel $\widetilde{H}(s, t)$. Then system of integral equations (5.4) and (5.5) has the unique solution $g_{1}(t) \in C[0, \beta]$ and $g_{2}(t) \in C[-\alpha, 0]$, and then the function $u(x, t)$ is found by formula (2.1). If condition (3.12) fails, system of integral equations (5.4) and (5.5) has a solution up to a term, whose factor is an unknown number $g_{1}(0)$ and the same is true for Problem 4.

Let us show that conditions $f_{1}\left(x_{0}\right) \neq 0$ or $f_{2}\left(x_{0}\right) \neq 0$ are essential for the unique solvability of Problem 4.

Let $f_{1}\left(x_{0}\right)=0$, and $f_{2}\left(x_{0}\right) \neq 0$. Then there exists a function $f_{1}(x)=\sin \mu_{m} x=\sin \pi n \widetilde{x}$, where $m$ is some fixed natural number, $\widetilde{x}=x / l$, such that $f_{1}\left(x_{0}\right)=\sin \pi m x_{0}=0$. For the function $f_{1}(x)=\sin \mu_{m} x$ and arbitrary $g_{1}(t) \in C[0, \beta], g_{2}(t) \in C[-\alpha, 0]$, there exists a non-zero solution of Problem 4 as $h_{1}(t) \equiv 0, h_{2}(t) \equiv 0$, which is determined by formula (3.13).

Let $f_{2}\left(x_{0}\right)=0$, and $f_{1}\left(x_{0}\right) \neq 0$. Then there exists a function $f_{2}(x)=\sin \mu_{n} x=\sin \pi n \widetilde{x}$, where $n$ is some fixed natural number, such that $f_{2}\left(x_{0}\right)=\sin \pi n \widetilde{x}_{0}=0$. Then for the function $f_{2}(x)=\sin \mu_{n} x$ and all $g_{1}(t) \in C[0, \beta], g_{2}(t) \in C[-\alpha, 0]$, there exits a non-zero solution of Problem 4 as $h_{1}(t) \equiv 0, h_{2}(t) \equiv 0$, which is determined by formula (4.24).

Let $f_{1}(x)=f_{2}(x)=\sin \mu_{m} x$, where $m$ is some fixed natural number be such that $f_{1}\left(x_{0}\right)=$ $f_{2}\left(x_{0}\right)=\sin \mu_{m} x_{0}=0$. Then for the functions $f_{1}(x)=f_{2}(x)=\sin \mu_{m} x$ and arbitrary $g_{1}(t) \in$ $C[0, \beta], g_{2}(t) \in C[-\alpha, 0]$ there exists a non-zero solution of Problem 4 as $h_{1}(t) \equiv 0, h_{2}(t) \equiv 0$ :

$$
u_{3 m}(x, t)=T_{3 m}(t) \sin \mu_{m} x
$$

where

$$
\begin{aligned}
& T_{3 m}(t)= \begin{cases}\frac{\omega_{3 m}(\alpha)}{\Delta_{m}(\alpha)} e^{-\lambda_{m}^{2} t}+\int_{0}^{t} g_{1}(s) e^{-\lambda_{m}^{2}(t-s)} d s, & t>0, \\
\frac{\omega_{3 m}(\alpha)}{\Delta_{m}(\alpha)} \Delta_{m}(-t)-\omega_{3 m}(-t), & t<0,\end{cases} \\
& \omega_{3 m}(-t)=g_{1}(0) \gamma_{\frac{1}{2 q}}(m) \sqrt{-t} J_{\frac{1}{2 q}}\left(p_{m}(-t)^{q}\right)-W_{m}(-t) .
\end{aligned}
$$

Similar to Section 3 we can show that function $(5.10)$ satisfies conditions $(1.2)-(1.9)$.

\section{BIBLIOGRAPHY}

1. I.M. Gel'fand. Some questions of analysis and differential equations // Uspekhi Matem. Nauk. 14:3, 3-19 (1959). (in Russian).

2. Ya. S. Uflyand. On oscillation propagation in compound electirc lines // Inzhener.-Phis. Zhurn. 7:1, 89-92 (1964). (in Russian). 
3. I.T. Lozanovskaya, Ya.S. Uflyand. A class of problems in mathematical physics with a mixed eigenvalue spectrum // Dokl. AN. SSSR. 164:5, 1005-1007 (1965). [Sov. Phys., Dokl. 10, 918-919 (1965).]

4. T.D. Dzhuraev, A. Sopuev, M. Mamazhanov. Boundary value problems for equations of parabolichyperbolic type. Izdat. "Fan" Uzbek. SSR, Tashkent (1986). (in Russian).

5. O.A. Ladyzhenskaya, L. Stupyalis. On mixed type equations // Vestnik LGU. Ser. Matem. Mekh. Astr. 19:4, 38-46 (1965). (in Russian).

6. L. Stupyalis. Initial-boundary value problems for equations of mixed type // Trudy MIAN SSSR. 27. 115-145 (1975). [Proc. Steklov Inst. Math. 127, 135-170 (1975).]

7. N.Yu. Kapustin. Tricomi problem for a parabolic-hyperbolic equation with degeneracy in the hyperbolic part. I. // Differ. Uravn. 23:1, 72-78 (1987). [Diff. Equat. 23:1, 51-55 (1987).]

N.Yu. Kapustin. Tricomi problem for a parabolic-hyperbolic equation with degeneracy in the hyperbolic part. II. // Differ. Uravn. 24:8, 1379-1386 (1988). [Diff. Equat. 24:8, 898-903 (1988).]

8. N.Yu. Kapustin, E.I. Moiseev. On the spectral problem from the theory of the parabolic-hyperbolic heat equation // Dokl. Akad. Nauk. Ross. Akad. Nauk 352:4, 451-454 (1997). [Dokl. Math. 55:1, 80-83 (1997).]

9. K.B. Sabitov. Direct and inverse problems for mixed parabolic-hyperbolic equations. Nauka, Moscow (2016). (in Russian).

10. K.B. Sabitov. Initial boundary and inverse problems for the inhomogeneous equation of a mixed parabolic-hyperbolic equation // Matem. Zamet. 102:3, 415-435 (2017). [Math. Notes. 102:3, 378395 (2017).]

11. K.B. Sabitov, L.Kh. Rakhmanova. Initial-boundary value problem for an equation of mixed parabolic-hyperbolic type in a rectangular domain // Differ. Uravn. 44:9, 1175-1181 (2008). [Diff. Equat. 44:9, 1218-1224 (2008).]

12. K.B. Sabitov. Tricomi problem for a mixed parabolic-hyperbolic equation in a rectangular domain // Matem. Zamet. 86:2, 273-279 (2009). [Math. Notes. 86:2, 249-254 (2009).]

13. K.B. Sabitov. Initial-boundary value problem for a parabolic-hyperbolic equation with power-law degeneration on the type change line // Differ. Uravn. 47:10, 1474-1481 (2011). [Diff. Equat. 47:10, 1490-1497 (2011).]

14. K.B. Sabitov, S.N. Sidorov. On a nonlocal problem for a degenerating parabolic-hyperbolic equation // Differ. Uravn. 50:3, 356-365 (2014). [Diff. Equat. 50:3, 352-361 (2014).

15. K.B. Sabitov, S.Sidorov. Initial-boundary-value problem for inhomogeneous degenerate equations of mixed parabolic-hyperbolic type // Itogi Nauki i Tekhniki. Ser. Sovrem. Mat. Pril. Temat. Obz. 137, 26-60 (2017). [J. Math. Sci. 236:6, 603-640 (2019).]

16. K.B. Sabitov, E.M. Safin. The inverse problem for a mixed-type parabolic-hyperbolic equation in a rectangular domain // Izv. VUZov. Matem. 56:4, 55-62 (2010). [Russian Math. Iz. VUZ. 54:4, 48-54 (2010).]

17. K.B. Sabitov, E.M. Safin. The Inverse problem for an equation of mixed parabolic-hyperbolic type // Matem. Zamet. 87:6, 907-918 (2010). [Math. Notes. 87:6, 880-889 (2010).]

18. K.B. Sabitov, S.N. Sidorov. Inverse problem for degenerate parabolic-hyperbolic equation with nonlocal boundary condition // Izv. VUZov. Matem. 1, 46-59 (2015). [Russian Math. (Iz. VUZ). 59:1, 39-50 (2015).]

19. M.M. Lavrent'ev, K.G. Reznitskaya, V.G. Yakhno. One-dimensional inverse problems of mathematical physics. Nauka, Novosibirsk (1982). (in Russian).

20. V.G. Romanov. Inverse problems of mathematical physics. Nauka, Moscow (1984). (in Russian).

21. V.G. Romanov, S.I. Kabanikhin. Inverse problems of geoelectricity. Nauka, Moscow (1991). (in Russian).

22. A.M. Denisov. Introduction in the theory of inverse problems. Moscow State Univ., Moscow (1994). (in Russian).

23. A.I. Prilepko, D.G. Orlovsky, I.A. Vasin. Methods for solving inverse problems in mathematical physics. Monograph and Textbooks in Pure and Applied Mathematics. 222. Marcel Dekker Inc, New York (2000).

24. V. Isakov. Inverse problem for partial differential equations. Springer, New York (2006). 
25. S.I. Kabanikhin. Inverse and ill-posed problems. Theory and applications. Sibir. knizh. izd-vo, Novosibirsk (2009). [de Gruyter, Berlin (2012).]

26. A.I. Prilepko, V.V. Solov'ev. Solvability theorems and Rothe's method for inverse problems for a parabolic equation. I // Differ. Uravn. 23:10, 1791-1799 (1987). [Diff. Equat. 23:10, 1230-1237 (1987).]

A.I. Prilepko, V.V. Solov'ev. Solvability theorems and Rothe's method for inverse problems for a parabolic equation. II // Differ. Uravn. 23:11, 1971-1980 (1987). [Diff. Equat. 23:11, 1341-1349 (1987).]

27. A.I. Prilepko, I.A. Vasin. Uniqueness of solutions in some nonlinear inverse problems of hydrodynamics // Differ. Uravn. 26:1, 109-120 (1990). [Diff. Equat. 26:1, 97-105 (1990).]

28. A.I. Prilepko, A.B. Kostin. Estimation of the spectral radius of an operator and the solvability of inverse problems for evolution equations // Matem. Zamet. 53:1, 89-94 (1993). [Math. Notes. 53:1, 63-66 (1993).]

29. V.V. Solov'ev. Finding the source and the coefficients of a parabolic equation in the multidimensional case // Differ. Uravn. 31:6, 1060-1069 (1995). [Differ. Equat. 31:6, 992-1001 (1995).]

30. V.V. Solov'ev. Global existence of a solution to the inverse problem of determining the source term in a quasilinear equation of parabolic type // Differ. Uravn. 32:4, 536-544 (1996). [Diff. Equat. 32:4, 538-547 (1996).]

31. A.B. Kostin. The inverse problem of recovering the source in a parabolic equation under a condition of nonlocal observation // Matem. Sborn. 204:10, 3-46 (2013). [Sb. Math. 204:10, 1391-1434 (2013).]

32. A.I. Kozhanov. A nonlinear loaded parabolic equation and a related inverse problem // Matem. Zamet. 76:6, 840-853 (2004). [Math. Notes. 76:6, 784- 795 (2004).]

33. A.I. Kozhanov, R.R. Safiullova. Linear inverse problems for parabolic and hyperbolic equations // J. Inver. Ill-Posed Probl. 18:1, 1-18 (2010).

34. V.I. Arnol'd. Small denominators. I. Mapping the circle onto itself // Izv. RAN. Ser. Matem. 25:1, 21-86 (1961). (in Russian).

35. A. Erdélyi, W. Magnus, F. Oberhettinger, F.G. Tricomi. Higher transcendental functions. V. 2. Robert E. Krieger Publ. Co., Malabar, Florida (1981).

36. K.B. Sabitov. Functional, differential and integral equations. Vysshaya Schkola, Moscow (2005). (in Russian).

Stanislav Nikolaevich Sidorov,

Sterlitamak branch of

Institute of Strategic Studies of RB,

Odesskaya str. 68,

453103, Sterlitamak, Russia,

Sterlitamak branch of Bashkir State Univeristy,

Lenin av. 37,

453103, Sterlitamak, Russia

E-mail: stsid@mail.ru 